\title{
The Effect of Parameters Variation on Bilateral Controller
}

\author{
Sari Abdo Ali ${ }^{1}$, Muhammad Fahmi Miskon ${ }^{2}$, Ahmad Zaki Hj Shukor ${ }^{3}$, Marwan Qaid Mhoammed ${ }^{4}$ \\ Center of Excellence in Robotic and Automation, Universiti Teknikal Malaysia Melaka, Malaysia
}

\begin{tabular}{l} 
Article Info \\
\hline Article history: \\
Received Mar 5, 2018 \\
Revised Mar 21, 2018 \\
Accepted Apr 5, 2018 \\
\hline
\end{tabular}

Keyword:

DC motor

Bilateral teleoperation

DOB/RTOB

Haptic control

Haptics

\begin{abstract}
This paper analyzes the effects of the bilateral control parameters variation on the stability, the transparency and the accuracy, and on the operational force that is applied to DC motor and the master system. The bilateral controller is designed for rehabilitation process. PD controller is used to control the position tracking and a force gain controller is used to control the motor torque. DOB eliminate the internal disturbance and RTOB to estimate the joint torque without using sensors. The system consists of two manipulators, each manipulator has 1dof, master and slave teleoperation system, 4 controlarchitecture channel, DOB and reaction force observer. The master system is attached to human oberator. The slave system is attached to external load. The aim in this paper is to design the controller so that it requires less force to move the master manipulator and at the same time achieve high performance in position tracking.
\end{abstract}

Copyright $(0) 2018$ Institute of Advanced Engineering and Science. All rights reserved.

\section{Corresponding Author:}

Sari Abdo Ali,

Center of Excellence in Robotic and Automation

Fakulti Kejuruteraan Elektrik Universiti Teknikal Malaysia Melaka.,

Hang Tuah Jaya, 76100 Durian Tunggal, Melaka, Malaysia.

Email: sary_ad@yahoo.com

\section{INTRODUCTION}

Haptic systems have been developed in the last decade to establish motion control with high performance. Haptic motion control systems are widely used in teleoperation where human machine interface is performed with the environment. Human machine interaction enables people to do tasks in hazardous environments such as nuclear plants, and under water without been exposed to danger. Teleoperation systems are classified to unilateral, bilateral and multilateral[1]. The focus is on bilateral teleoperation because it has two ways data communication, from the person to machine to the environment, then feedback the response to the person. It is expected to play important role for the upcoming generation of robotic devices. The bilateral system enables the user to feel the reaction from the environment. There are challenges affect the design of the haptic bilateral controller and the performance such as transparency, the stability, the operationality and producibility[2].

Controlling a motor motion usually is done by controlling the speed or by controlling the position. The type of controller depends on the motor application and the desired motion wherther it is a AC or DC motor. In [3] [4] a PI controller is used to control the motor speed because it produce more accurate result than a PD. However, in this paper a PD controller is used. PD controller shows more accurate result when integrated with bilateral haptic controller due to the effect of the disturbance observer. If PI is used in bilateral controller the motor will be unstable.

The bilateral controller consists of common mode that deals with torque of both master and slave and differential mode that handle the position as figure 1 illustrate[5]. The figure shows the four-channel architecture of bilateral control. The bilateral teleoperation requires position control and force control. Both position and force control have different dimensions and con not be controlled together at the same time [6]. For that reason, the acceleration-based method is introduced to control them at one dimension which is the acceleration dimension. Both can be represented in terms of acceleration. The acceleration-based method can 
be robust by proper design of the DOB[7]. The disturbance observer estimates the disturbance in the system and improve the stability. There are two proposed DOB; low pass filter DOB and high pass filter DOB [8]. The higher DOB improve the system performance, suppress disturbance, performs better with low sampling controller's rates. However, the robustness is reduced for wide bandwidth range. The low DOB is first order low pass filter that improve the robustness of the system but reduce the performance. The dynamics characteristic of low DOB results inefficient bandwidth.

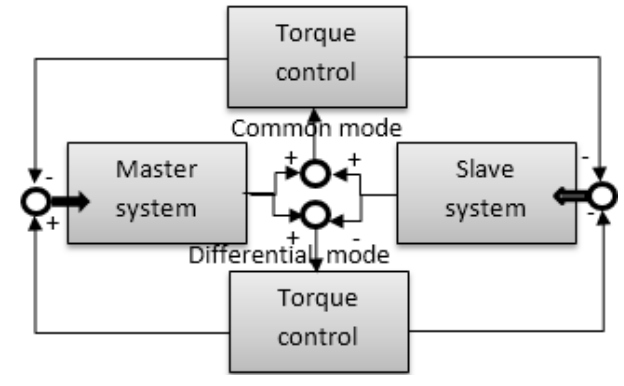

Figure 1. The Four-Channel Architecture of Bilateral Control

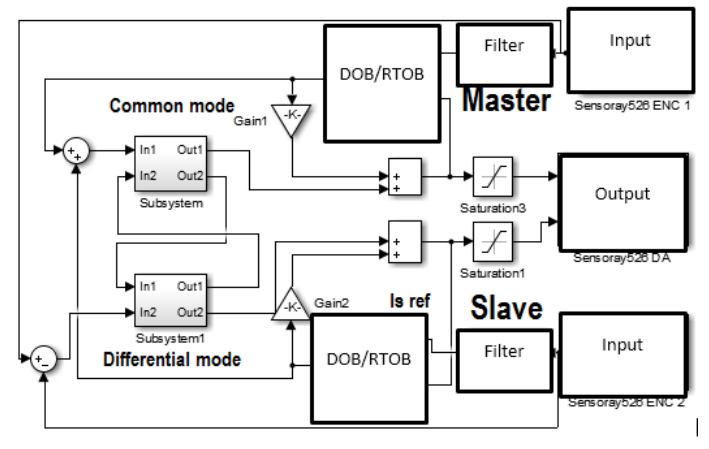

Figure 2. Bilateral Control Block Diagram

The RTOB is used to estimate reaction force from the environment. RTOB is used to replace the force sensors[9]. Force sensors because sensors have narrow bandwidth and accompanied with noise that make the system unstable. The design of DOB and RTOB effect the stability and the transparency[10], while the accuracy of the system position tracking is affected by the PD. The block diagram of the haptic bilateral system is shown in figure two. The DOB output improves the inner loop performance and the RTOB improves the outer loop performance. Basically, the system works as follow: the master system motion is detected by the encoder. The signal is sent from the encoder to differentiator that derive the velocity. This step produce noise. Then, the signal is sent to low pass filter to eliminate the noise produced by the differentiator. The velocity is sent to DOB and the position is sent to the differential mode. the force is derived and sent to the common mode. the slave motor moves the same motion produced by the master motor. any interaction with the environment by the slave manipulator will be reflected back to the motor of the master and will be felt by the operator.

This paper investigates the effects of the parameters variation of bilateral controller on the stability, the transparency and the accuracy. Based on the investigation, we want to select the suitable parameters that result lower operation force at the master manipulator. At the same time keeping the accuracy of the position tracking between the master and the slave manipulators. The paper is organized as follow: section 1 is a brief introduction of the bilateral haptic controller. Section 2 discusses the methodology, the setup of the experiment and the mathematical representation of bilateral control. Section 3 is about the experiment results and discussion. Section 4 is a conclusion and future work.

\section{RESEARCH METHOD}

The prototype hardware of the experiment is shown in Figure 3 and it requires a geared DC-Motor with installed encoder on it, micro-box, motor driver and power supply. Micro-box is real time controller that is used to interface the hardware module with programming host (e.g. computer). The advantages micro box is that it has high performance, consume low power, small size, AD/DA, Encoder and DI/O modules. The power supply used can deliver 3 output types: $+5 \mathrm{~V} / 10 \mathrm{~A},+15 \mathrm{~V} / 3.5 \mathrm{~A}$ and $15 \mathrm{~V} / 1 \mathrm{~A}$. Two systems are used in the experiment. the same components are used in both systems. A DC-motor is used as an actuator. An attached gear head to the motor is to increase the torque of the output by a ratio of $36: 1$. The motor has an encoder that has 5000 pulse/rev. The motor driver is needed to make the micro-box output current suitable for the motor. The current delivered by the micro box is not enough to activate the geared DC-motor.

The overall working principle as shown in figure 3 is that the master motor is actuated by external force which called operational force applied by a human. The aim of the hardware is to analyze the factors that enable us to use less operation force to move the slave manipulator 


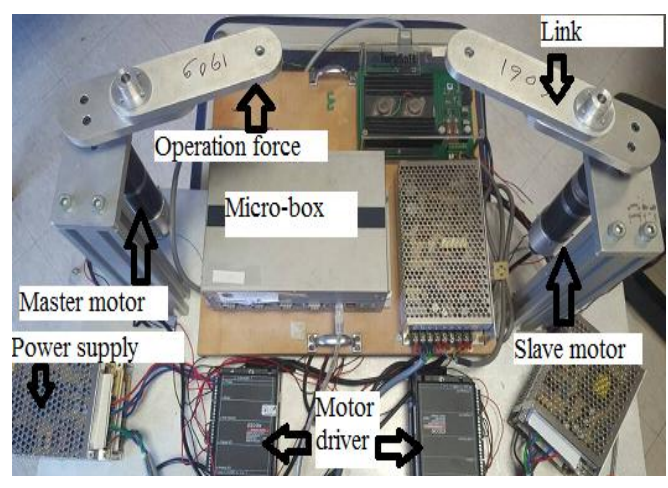

Figure 3. The Experimental Hardware Setup

\subsection{Bilateral Controller}

Bilateral control system is based on acceleration-based method and has two modes, common mode and differential mode. Differential mode tracks the position between master and slave motors (as in equation 1). The ideal tracking should result zero. A common mode is to control the force from the master motor and copy it to the slave motor as in equation 2 .

$$
\begin{aligned}
& \theta=\theta_{m}-\theta_{s}=0 \\
& \tau=\tau_{m}+\tau_{s}=0 \\
& \ddot{\theta}_{D i f}=-c_{p}\left(\theta_{m}-\theta_{s}\right) \\
& \ddot{\theta}_{c o m}=-c_{p}\left(\tau_{m}+\tau_{s}\right) \\
& c_{p}=k_{p}+k_{d} s
\end{aligned}
$$

$\mathrm{Cp}$ in equation 3 and 4 is for the PD controller in equation 5. It can be written as in 6.

$$
u(t)=k_{p}\left(\theta_{r e f}-\dot{\theta}\right)+k_{d}\left(\dot{\theta}_{r e f}-\dot{\theta}\right)
$$

The PD design is built and tested based on the requirement of motor parameters. The overall model of the PD is explained in[11].

\subsection{Disturbance Observer}

DOB compensates the disturbance forces of the motor in the inner loop of the bilateral controller and estimates it. DOB is design is shown in the following equations. The parameters definitions used in the paper are as follow:
J Actual inertia
Jn the nominal inertia
Acceleration of the motor;
Mechanical torque;
Load torque
External torque applied on the system
Internal torque
Coulomb friction
Viscous torque
Disturbance torque
Torque constant
Current flow in the motor. 
The motor torque is represented in 7

$$
\begin{aligned}
& \mathrm{J} \ddot{\theta}=\tau_{m e c}-\tau_{l} \\
& \tau_{l}=\tau_{\text {ext }}+\tau_{\text {int }}+\tau_{c o}+D \dot{\theta} \\
& \tau_{m e c}=k_{t} \mathrm{I} \\
& \mathrm{J} \ddot{\theta}=k_{t} \mathrm{I}-\left(\tau_{\text {ext }}+\tau_{\text {int }}+\tau_{c o}+D \dot{\theta}\right)
\end{aligned}
$$

The disturbance force can be represented as

$$
\tau_{d i s}=-\Delta k_{t} \mathrm{I}+\tau_{l}+\Delta \mathrm{J} \ddot{\theta}
$$

the center of mass changes over time because of configuration of the mechanical system which change the inertia $\mathrm{J}$ as well.

$$
\begin{aligned}
& \mathrm{J}=J_{n}+\Delta J \\
& k_{t}=k_{t n}+\Delta k_{t}
\end{aligned}
$$

Applying equations $12 \& 13$ in 10

$$
\begin{aligned}
& \left(J_{n}+\Delta J\right) \ddot{\theta}=\left(k_{t n}+\Delta k_{t}\right) \mathrm{I}-\left(\tau_{\text {ext }}+\tau_{\text {int }}+\tau_{c o}+D \dot{\theta}\right) \\
& \operatorname{Or}\left(J_{n}+\Delta J\right) \ddot{\theta}=\left(k_{t n}+\Delta k_{t}\right) \mathrm{I}-\tau_{l}
\end{aligned}
$$

Reorganize 11 and 14 equation we get

$$
J_{n} \ddot{\theta}=\left(k_{t n} \mathrm{I}-\tau_{d i s}\right) \text { Or } \tau_{d i s}=\left(k_{t n} \mathrm{I}-J_{n} \ddot{\theta}\right)
$$

Equation 15 is the estimated torque in the DOB. However, the torque estimated in DOB is filtered as in equation 16. Gdob is the Cut-Off frequency.

$$
\tau^{\wedge}{ }_{e x t}=\left(k_{t} \mathrm{I}-\mathrm{J} \dot{\theta}\right) \frac{G_{D O B}}{s+G_{D O B}}
$$

\subsection{Reaction Torque Observer}

RTOB and DOB have similar structure RTOB replace the use of sensors in bilateral controller. RTOB is DOB application. It estimates the impedance of the environment or in other words estimate the reaction force in the motor that is created by interaction with the environment. The reaction force is estimated by equation 18

$$
\begin{aligned}
& \tau_{\text {ext }}=\tau_{\text {dis }}-\tau_{\text {int }}-\tau_{c o}-D \dot{\theta} \\
& \tau_{\text {ext }}^{\wedge}=\left(k_{t} \mathrm{I}-\mathrm{J} \dot{\theta}+\tau_{\text {ext }}\right) \frac{G_{\text {RTOB }}}{s+G_{R T O B}} \\
& \tau_{\text {ext }}^{\wedge}=\tau_{\text {ext }} \cdot \frac{G_{R T O B}}{s+G_{R T O B}}
\end{aligned}
$$

The initial parameters of the experiment are shown in table 1 it shows that some values will be changed, depend on the experiment. The mass of the links, the inertia, the length the gear ratio and torque's constant will not be changed throughout all experiments. 
Table 1. The Experiment Parameters

\begin{tabular}{cc}
\hline Parameter & Value \\
\hline Torque constant & $0.7164 \mathrm{~N} \backslash \mathrm{A}$ \\
Inertia & $0.120 \mathrm{~kg} . \mathrm{cm} 2$ \\
Gear ratio & $36: 1$ \\
Cut-off frequency & 100 \\
Cut-off frequency of RTOB & 100 \\
Force gain & 2 \\
Link mass & $0.2 \mathrm{~kg}$ \\
Link length & $0.15 \mathrm{~m}$ \\
PD parameters & $\mathrm{Kp}=300, \mathrm{Kd}=30$ \\
\hline
\end{tabular}

\section{RESULTS AND ANALYSIS}

In this section, it explain the results of research and at the same time is given the comprehensive discussion. Results is presented in figures, graphs and tables

\subsection{The effects of DOB \& RTOB}

The first experiment is to analyze the DOB cut off frequency variation impact on the bilateral controller performance. The DOB estimate the disturbance in the inner loop of the bilateral controller and compensate it. The DOB does not affect or drive the controller toward instability. But it effects the operational force, the transparency smoothness of the motion. Figures 4 to 7 shows the effects and the changes of position tracking and force of the bilateral controller as the cut off frequency of the DOB is increased from 50, 100, 200, 500 .
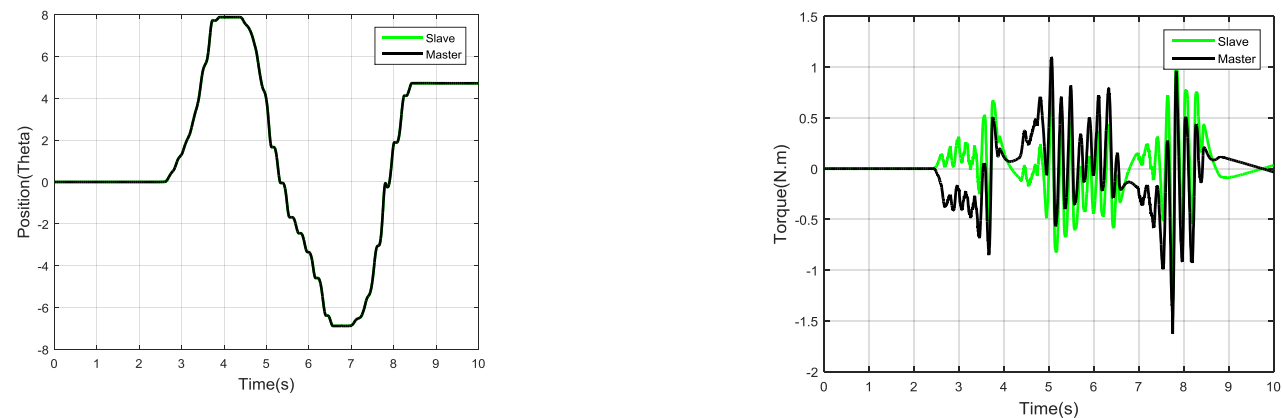

Figure 4. Position and Torque Response with DOB Frequency $=50$
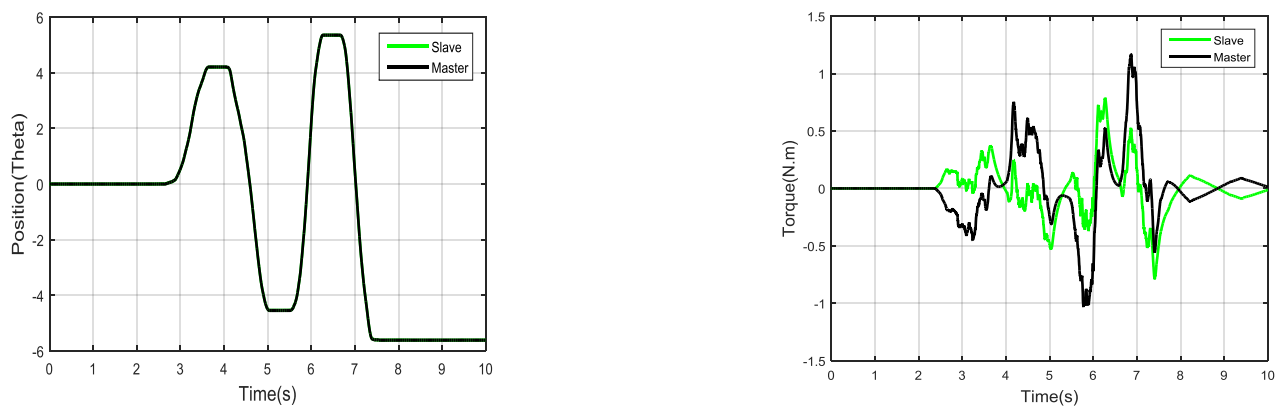

Figure 5. Position and Torque Response with DOB Frequency $=100$ 

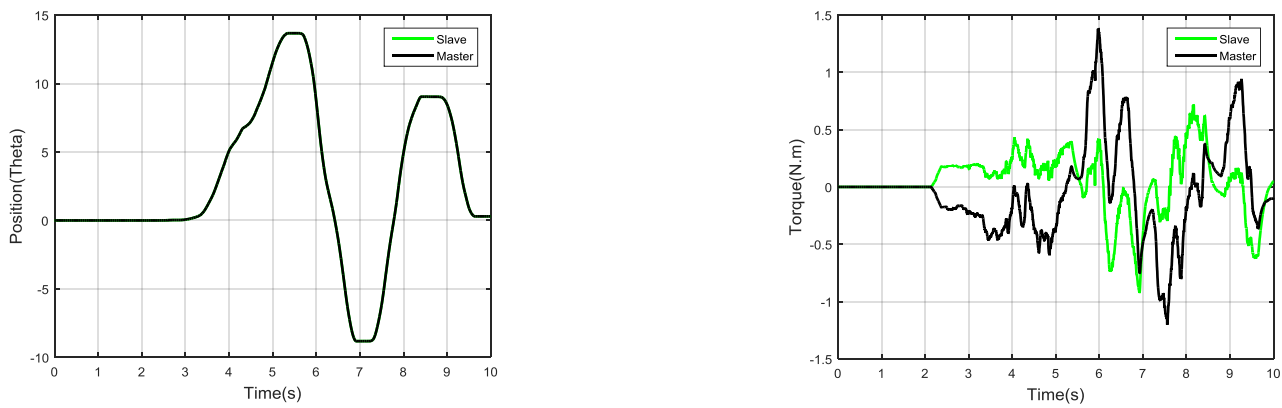

Figure 6. Position and Torque Response with DOB Frequency $=200$
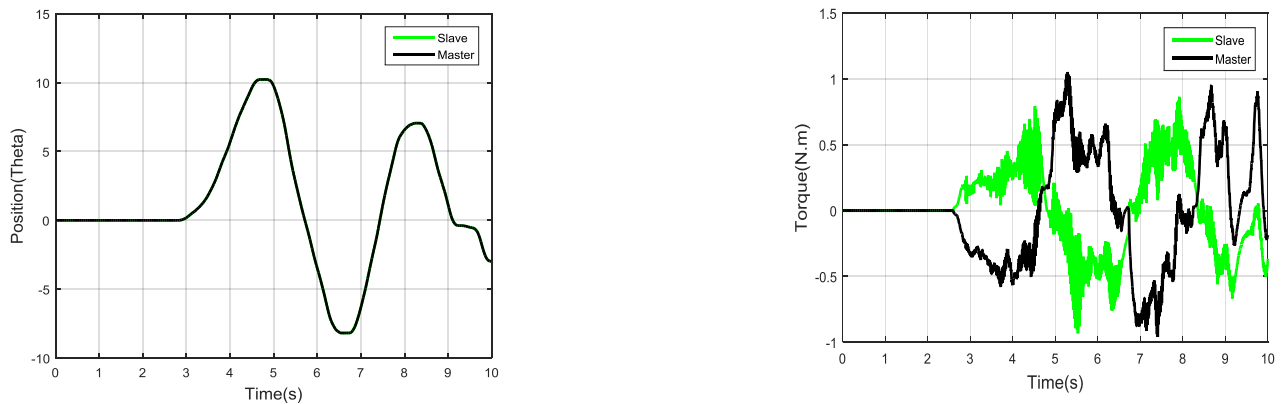

Figure 7. Position and Torque Response with DOB Frequency $=500$

The gained results in Figure 4 shows the Position and torque response with DOB frequency $=50$. The position response as illustrated is not smooth. In the real motion it result shaking in the motor link. However, the error tracking between the master and the slave is small even though the motion is not smooth. As the Gdob is increased the motion become smoother and the error between the master position and the slave position is reduced as shown in table II. However, the torque is affected negatively. As we can see that the transparency is reduced. A good transparency means that the mater torque is fully reproduced by the slave motor. there are a few reasons for this impact. The first graph shows a good transparency because Gdob is less than Grtob in table I. When GDOB is larger than GRTOB the noise is increased. Table 2 shows the error in the position and the force between the master and the slave manipulators.

Table 2. The Parameters of DOB Frequency

\begin{tabular}{ccc}
\hline Dob & Torque error & Position error \\
\hline 50 & 0.2806 & 0.0057 \\
100 & 0.2831 & 0.0034 \\
200 & 0.4672 & 0.0025 \\
500 & 0.6243 & 0.0020 \\
\hline
\end{tabular}

The second experiment is to vary the reaction torque parameters while the DOB and the other parameters remain as shown in table I. the results are shown in figures $8,9,10$. Table 3 shows the results in terms of error data as the parameters of RTOB frequency change from 50 to 500

Table 3. The Parameters of DOB Frequency

\begin{tabular}{ccc}
\hline RTOB & Torque error & Position error \\
\hline 50 & 0.4200 & 0.0057 \\
100 & 0.2842 & 0.0071 \\
200 & 0.4244 & 0.0045 \\
500 & 0.4400 & 0.0025 \\
\hline
\end{tabular}


The third experiment is to change both the DOB and the reaction torque parameters. The other parameters remain as in Table 1 . The results are shown in figures $11,12,13$. Table 4 shows the results for increasing parameters of DOB and RTOB, the frequency change from 50 to 500. The best results are gained when both DOB \& RTOB increased together to 100 with position error of 0.001

Table 4. Result of Increase DOB\&RTOB

\begin{tabular}{ccc}
\hline Dob \& RTOB & Torque error & Position error \\
\hline 50 & 0.2806 & 0.0057 \\
100 & 0.2501 & 0.0012 \\
200 & 0.4303 & 0.0026 \\
500 & 5.99 & 0.0432 \\
\hline
\end{tabular}
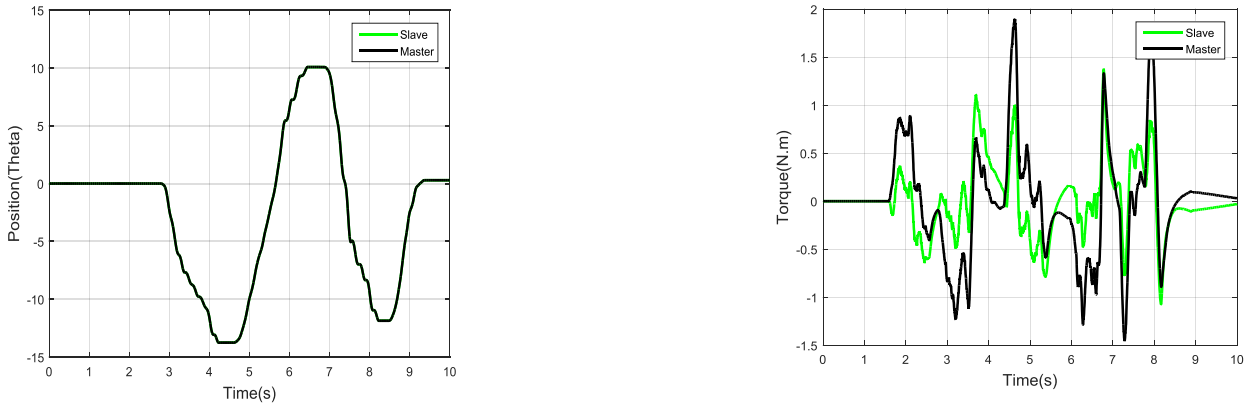

Figure 8. Position and Torque Response with RTOB Frequency $=50$
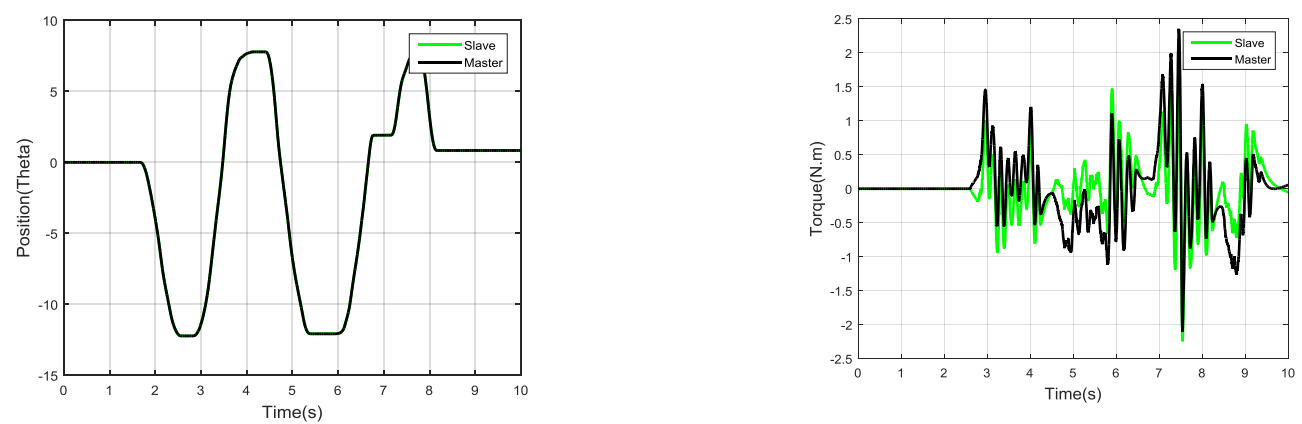

Figure 9. Position and Torque Response with RTOB Frequency $=100$
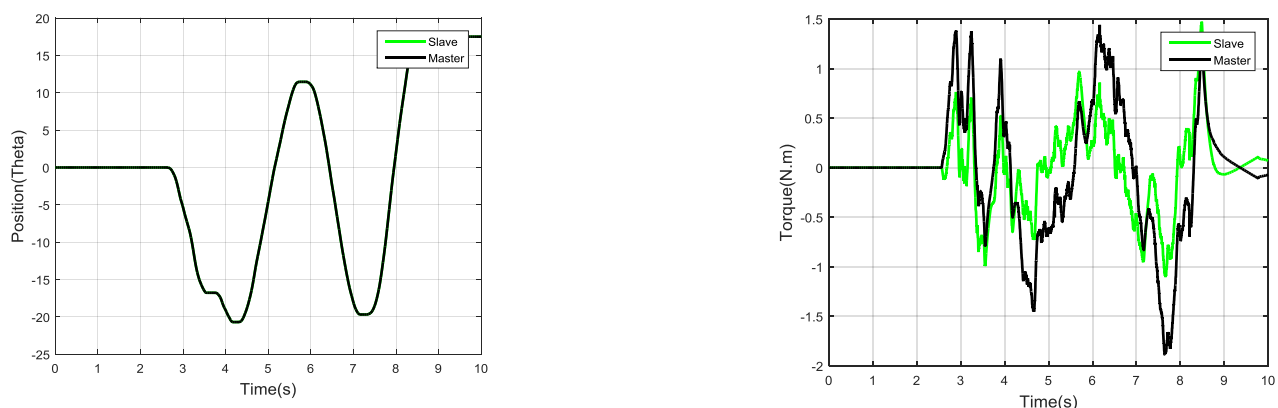

Figure 10. Position and Torque Response with RTOB Frequency $=200$ 
Briefly, the results in Figure 8 is similar to Figure 4 in terms of tracking Position (both have 0.0057 error). However, Figure 4 and 8 shows different torque response with frequency $=50$. The torque error in figure 4 is 0.2806 and in figure 8 is 0.4200 . this is because of the value of RTOB frequency is less than DOB frequency As the GDOB is increased more than GRTOB, the transparency is deteriorated and the error between the master force and the slave 655orce is increased as shown in table II and III. The transparency is improved when the cut off frequency of the DOB and RTOB are equal (have the same values). This is shown is Figures 11 and 12. Not only the transparency is improved, the position tracking improved as well. the increasing of DOB and RTOB frequency is limited and cannot be increased more than 500 because of the sampling time of the micro-box. Figure 13 illustrate a lot of unwanted noise which make the force controller unstable. The operational force required to move the master manipulator is less when both DOB and RTOB frequencies are equal.
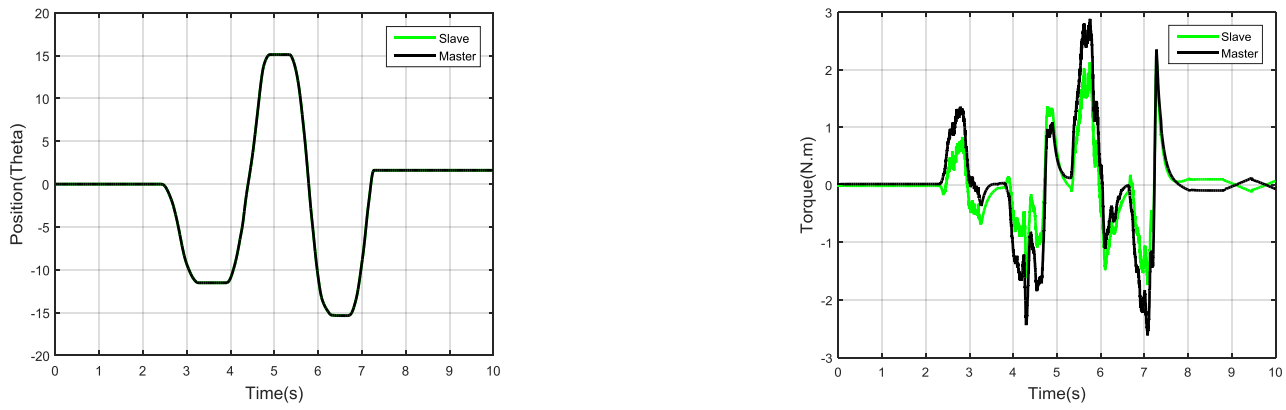

Figure 11. Position and Torque Response with both DOB and RTOB Frequency $=100$
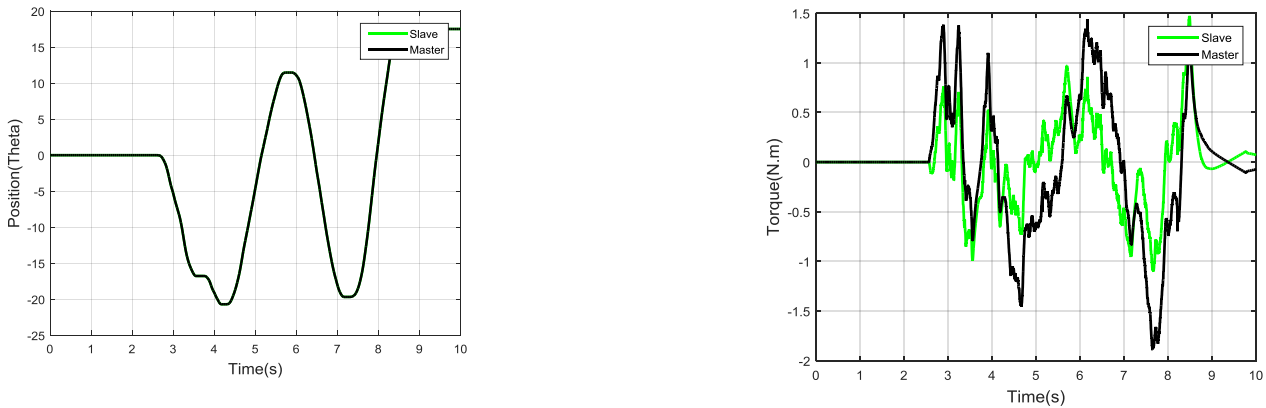

Figure 12. Position and Torque Response with both DOB and RTOB Frequency $=200$
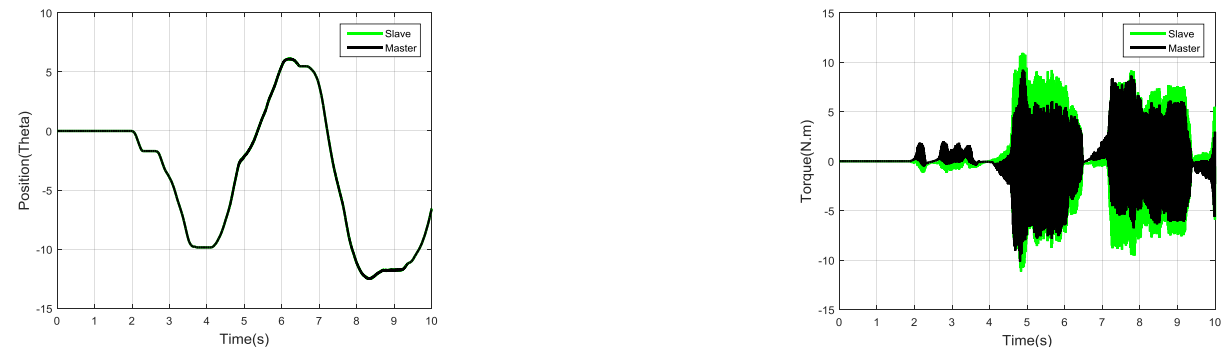

Figure 13. Position and Torque Response with both DOB and RTOB Frequency $=500$

\subsection{The effects of $P D$ parameters and force gain}

This section analysis the effects of changing the PD controller parameters and the force gain on the bilateral control response. The PD values that are tested and the torque gains are shown in Table 5. The values of the PD are selected based on the DC motor model that is built to check the response of the 
parameters. If the model is stable, then the value is used if not it is eleiminated. The motor model is discussed in previous experiment[11].

Table 5: PD and Force Gain Parameters

\begin{tabular}{cc}
\hline Parameter & Value \\
\hline PD Case 1 & $\mathrm{Kp}=0.5, \mathrm{Kd}=0.09$ \\
PD Case 2 & $\mathrm{Kp}=5, \mathrm{Kd}=0.1$ \\
PD Case 3 & $\mathrm{Kp}=50, \mathrm{Kd}=1$ \\
PD Case 4 & $\mathrm{Kp}=200, \mathrm{Kd}=20$ \\
PD Case 5 & $\mathrm{Kp}=300, \mathrm{Kd}=30$ \\
Force gain 1 & 0.5 \\
Force gain 2 & 1 \\
Force gain 3 & 2 \\
Force gain 3 & 5 \\
\hline
\end{tabular}

The PD consists of Kp gain and Kd gain. Section A shows that DOB and RTOB have effects on transparency and have not much effects on position tracking. The position tracking is more controlled be the PD controller. The first parameters of the PD $(\mathrm{Kp}=0.5$ and $\mathrm{Kd}=0.01)$ are selected based on root locus simulation model. These values suit the simulation, but with the real hardware application the values result error in the tracking. The motion with these values is stable. Figure 14 shows stable smooth motion with error of 0.67 as Table 6 shows.

Table 6. PD Parameters Variation Effects

\begin{tabular}{ccc}
\hline PD parameter $(\mathrm{Kp}, \mathrm{Kd})$ & Torque error & Position error \\
\hline $0.5,0.09$ & 0.5737 & 0,6759 \\
$5,0.1$ & 0.5563 & 0.7063 \\
50,1 & 0.5140 & 0.0606 \\
200.20 & 0.5926 & 0.0157 \\
300,30 & 0.3074 & 0.0041 \\
\hline
\end{tabular}
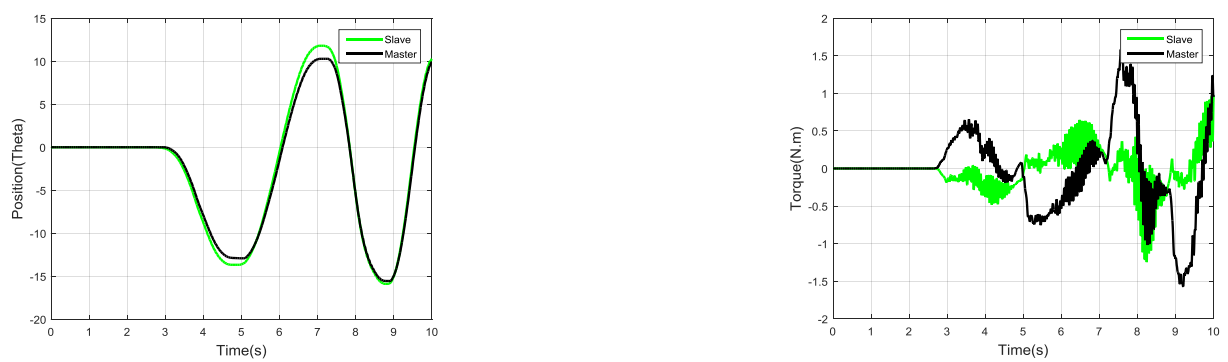

Figure 14. Position and Torque Response with $\mathrm{Kp}=0.5$ \& $\mathrm{Kd}=0.09$
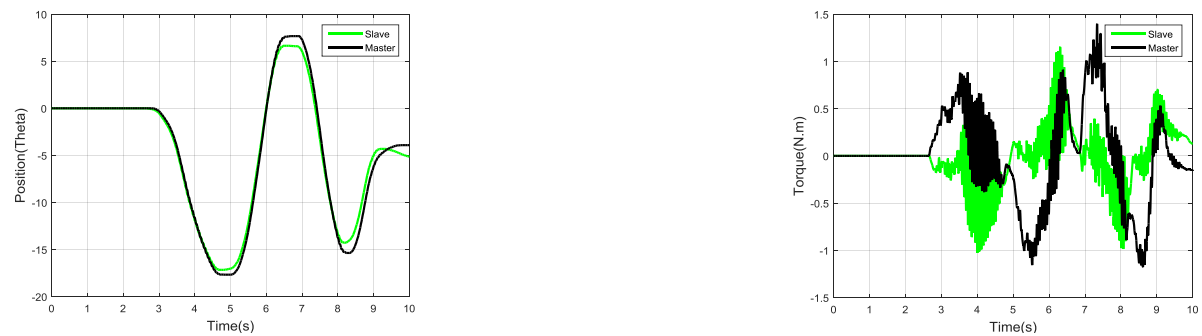

Figure 15. Position and Torque Response with $\mathrm{Kp}=5 \& \mathrm{Kd}=0.1$ 

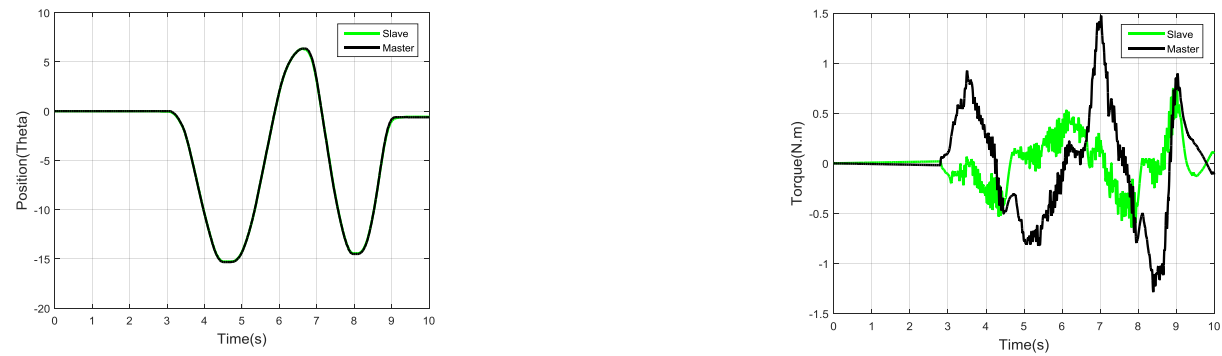

Figure 16. Position and Torque Response with $\mathrm{Kp}=50 \& \mathrm{Kd}=1$
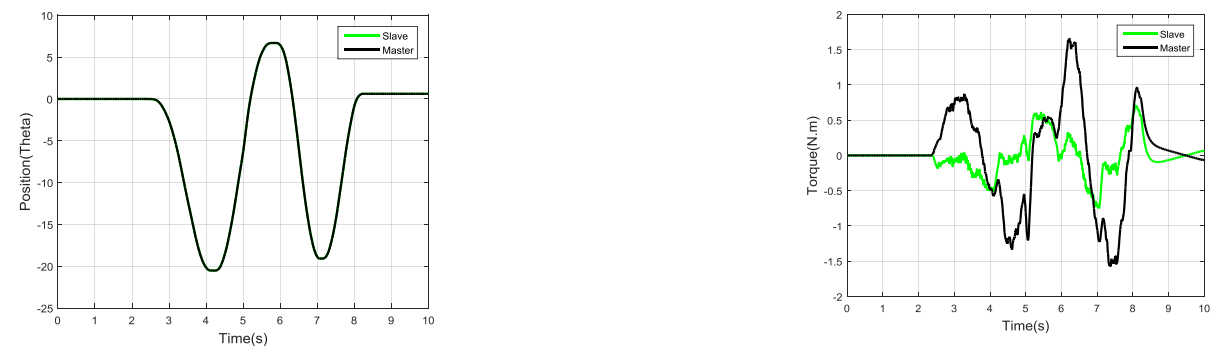

Figure 17. Position and Torque Response with $\mathrm{Kp}=200 \& \mathrm{Kd}=20$
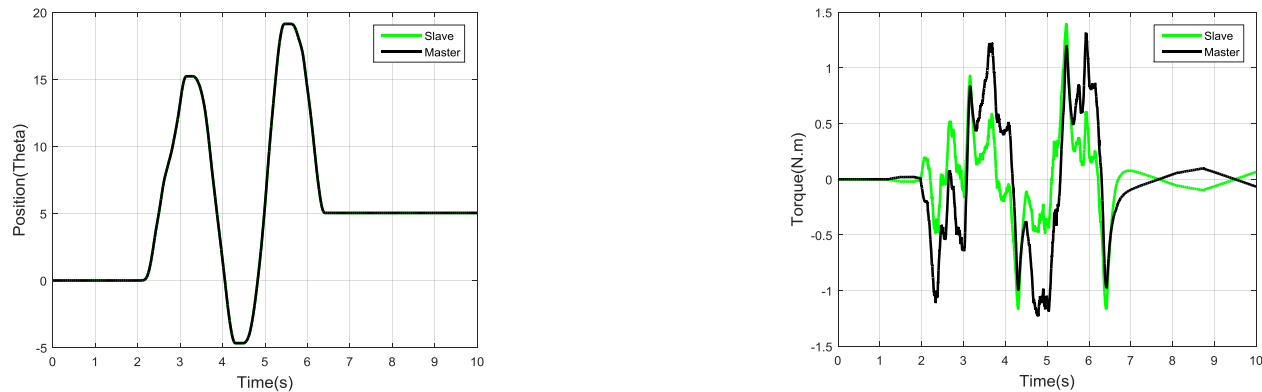

Figure 18. Position and Torque Response with $\mathrm{Kp}=300 \& \mathrm{Kd}=30$

As the results in the figures shown, if the values of the Pd gains increase, the position tracking between the master and the slave improve. The performance of the system improved too. The improvement is clearly appeared from Figure 14 to Figure 18. The force gain variation influences the stiffness and the operation force of the motor. The force gain does not have remarkable influence on position tracking. Increasing the force gain improve the transparency and the stability. Table 7 shows the results of increasing the force gain and Figures 19, 20 and 21 illustrate the response.

Table 7. Force Gain Variation Effects

\begin{tabular}{lll}
\hline Force gain & Torque error & Position error \\
\hline 0.5 & 0.6562 & 0.0045 \\
1 & 0.9235 & 0.0088 \\
2 & 0.3703 & 0.0043 \\
5 & 32.8717 & 0.9 \\
\hline
\end{tabular}




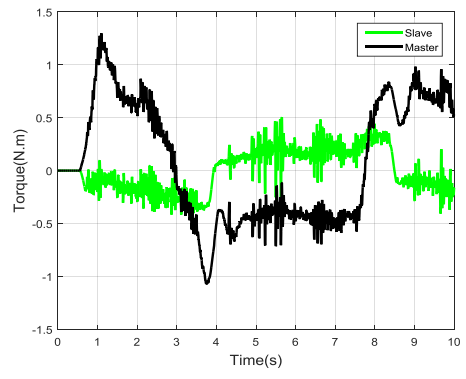

Figure 19. Position and Torque Response with Force Gain $=0.5$
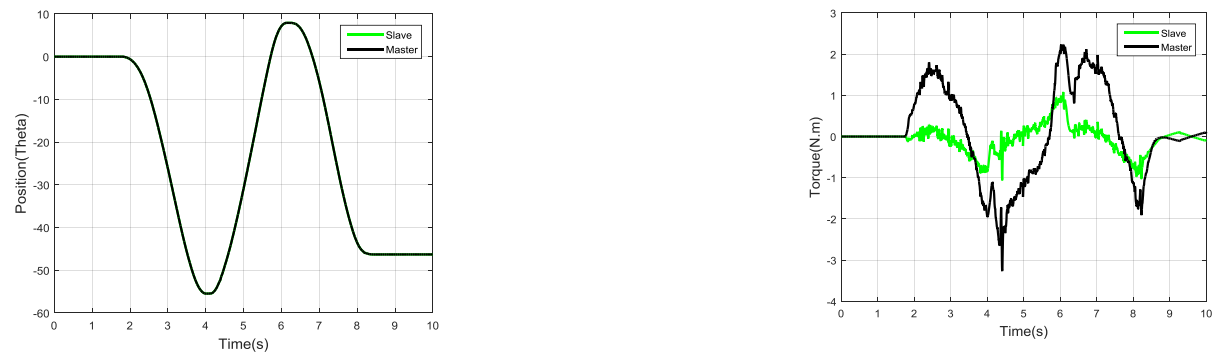

Figure 20. Position and Torque Response with Force Gain=1
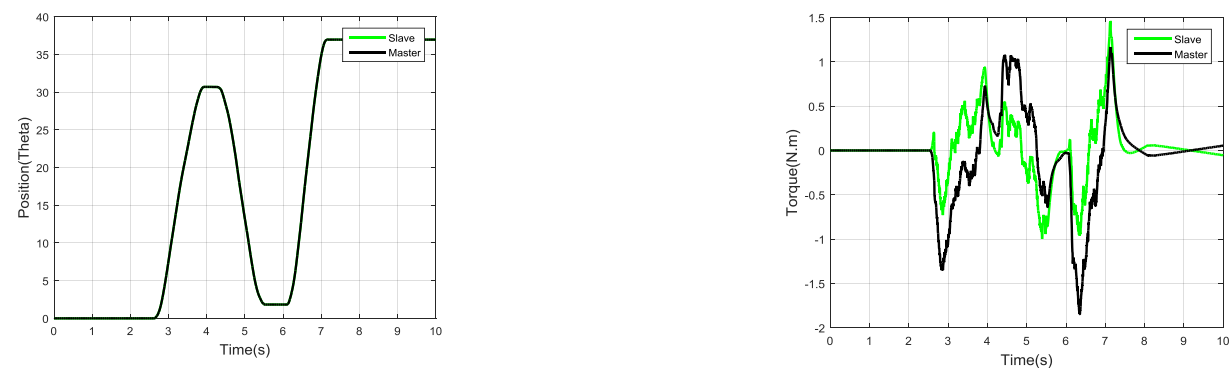

Figure 21. Position and Torque Response with Force Gain=2

\section{CONCLUSION}

The bilateral controller purpose is to copy the position and force from the master system to the slave system. The important factors of a bilateral system are stability, transparency and accuracy. The results of the paper showed and analyzed the effects of the bilateral control parameters variation on these tree factors as well as the operation force required to move the motors. The input that is used to move the master manipulator is from human leg motion (e.g. not unit step or sinusoidal inputs).

The result showed that the bilateral controller accuracy in tracking the position is significantly affected by the PD parameters. The DOB effect the transparency of the system and effect the stability when its cut off frequency is larger than the RTOB. The RTOB design impact the accuracy the stability and the operational force required to move the master manipulator. The operational force is also influenced by the force gain. For future work, it is recommended to study the effects of fast and slow motion on the accuracy of the bilateral controller. The bilateral controller perform better with fast).

\section{ACKNOWLEDGEMENTS}

The authors would like to thank Center of Excellence in Robotic and Automation, Fakulti Kejuruteraan Elektrik Universiti Teknikal Malaysia Melaka. Hang Tuah Jaya, 76100 Durian Tunggal, Melaka, UTeM Zamalah Scheme and Ministry of Higher Education Malaysia, for supporting this project. 


\section{REFERENCES}

[1] S. A. Ali, M. F. Miskon, A. Zaki, H. Shukor, M. B. Bahar, and M. Q. Mohammed, "Review on Application of Haptic in Robotic Rehabilitation Technology," Int. J. Appl. Eng. Res. ISSN, vol. 12, no. 12, pp. 973-4562, 2017.

[2] M. Mihelj and J. Podobnik, Haptics for Virtual Reality and Teleoperation, vol. 64. 2012.

[3] S. Jezernik, G. Colombo, and M. Morari, "Automatic gait-pattern adaptation algorithms for rehabilitation with a 4DOF robotic orthosis," IEEE Trans. Robot. Autom., vol. 20, no. 3, pp. 574-582, 2004.

[4] M. Jannati, A. Monadi, N. Rumzi, N. Idris, M. Junaidi, and A. Aziz, "Speed Sensorless Vector Control of Unbalanced Three-Phase Induction Motor with Adaptive Sliding Mode Control," vol. 4, no. 3, p. 8694, 2014.

[5] K. Ohnishi, S. Katsura, and T. Shimono, "Motion Control for Real-World Haptics," IEEE Ind. Electron. Mag., vol. 4, no. 2, pp. 16-19, 2010.

[6] T. Okura and S. Katsura, "Simplified haptic walking system based on bilateral control," in International Workshop on Advanced Motion Control, AMC, 2010, pp. 222-227.

[7] K. Ohnishi, M. Shibata, T. Murakami, and I. Paper, "Motion control for advanced mechatronics," IEEE/ASME Trans. Mechatronics, vol. 1, no. 1, pp. 56-67, 1996.

[8] E. Sariyildiz and K. Ohnishi, "On the robustness of disturbance observer," in 2014 IEEE 13th International Workshop on Advanced Motion Control (AMC), 2014, pp. 31-36.

[9] S. Katsura, Y. Matsumoto, and K. Ohnishi, "Modeling of force sensing and validation of disturbance observer for force control," IEEE Trans. Ind. Electron., vol. 54, no. 1, pp. 530-538, 2007.

[10] D. A. Lawrence, "Stability and Transparency in Bilateral Teleoperation," IEEE Trans. Robot. Autom., vol. 9, no. 5, pp. 624-637, 1993.

[11] S. A. Ali, M. F. Miskon, A. Z. Hj Shukor, and M. Q. Mohammed, "Single link bilateral haptics control with PD controller and geared DC-motor in robotic rehabilitation technology," Int. J. Mech. Mechatronics Eng., vol. 17, no. 5, pp. 148-155, 2017. 